\title{
A 2-stage Distal Femur Reconstruction with Expandable Endoprosthesis in a Pediatric Patient Presenting with a Pathologic Fracture through an Osteosarcoma
}

\author{
Jasson Louie R. Arcinue, ${ }^{1}$ Edward H.M. Wang ${ }^{1}$ and Gregorio Marcelo S. Azores ${ }^{1}$ \\ ${ }^{1}$ Department of Orthopedics, College of Medicine and Philippine General Hospital University of the Philippines Manila
}

\begin{abstract}
Osteosarcoma in pediatric patients has traditionally been treated with amputation, especially if there is a pathologic fracture through the tumor.

We report the case of a 12-year-old boy who sustained a pathologic fracture through distal femoral osteosarcoma. After neoadjuvant chemotherapy, he underwent limb saving surgery: wide excision of the osteosarcoma followed by a second-stage reconstruction with an expandable tumor endoprosthesis. He has a functional score of $93 \%$ and is free of disease 8 years since diagnosis and 2 years since the end of lengthening.
\end{abstract}

This is the first reported case in Philippine medical literature of limb saving surgery in osteosarcoma reconstructed with an expandable endoprosthesis.

Key Words: Osteosarcoma, Expandable tumor endoprosthesis, Limb salvage surgery

\section{INTRODUCTION}

Corresponding author: Edward HM Wang, MD, MSc

Department of Orthopedics

Philippine General Hospital

University of the Philippines Manila

Taft Avenue, Manila 1000, Philippines

Telephone +632 5548400 local 6300

Email: ehwang@up.edu.ph
Osteosarcoma in pediatric patients has traditionally been treated with amputation, especially if there is a pathologic fracture through the tumor. The decision to undertake limb salvage surgery is confronted not only with oncologic issues of tumor recurrence but also with leg length and girth equalization while optimizing extremity function. These difficulties are made more challenging in an environment of meager resources and a situation where expandable tumor endoprostheses are not readily available. This case is being reported to highlight three distinctive points.

This is the first reported case in the Philippines of a completed expandable endoprosthesis reconstruction (EPR) with a follow-up of 8 years since diagnosis and oncologic treatment and 2-1/2 years since the end of lengthening. Secondly, this case highlights the success of a two-stage surgery (limb salvage with an improvised nail and bone cement followed by a second surgery for EPR reconstruction) contrary to the usual literature-reported practice of a single stage resection and reconstruction. Thirdly, the initial presentation of a pathologic fracture did not adversely affect the oncologic outcome after limb salvage surgery. 


\section{CASE REPORT}

A 12-year-old male presented with a fracture of his left femur after falling from a standing height. On plain radiographs, the distal femur was lytic and moth-eaten and MRI showed a soft tissue mass within the area of fracture (Figure 1A and 1B). Open biopsy revealed classic intramedullary high grade chondroblastic osteosarcoma, Broders grade III. Staging workup was normal and the patient's case was classified as Enneking IIB. Neo-adjuvant chemotherapy was started using the hospital standard European Osteosarcoma Intergroup 2 drug protocol of doxorubicin and cisplatinum with a total of 3 cycles preoperatively. There was fracture healing, albeit shortened and angulated, and clinical response to chemotherapy was good. After several meetings with the family, limb saving surgery was chosen over amputation even if the patient had not yet achieved skeletal maturity.

In December of 2008 (5 months post-biopsy), wide tumor excision was undertaken. The vastus intermedius and the distal portion of the vastus lateralis were removed with tumor while saving the rectus femoris, the proximal portion of the vastus lateralis and the entire vastus medialis muscle. Bone was cut at $24 \mathrm{~cm}$ from knee joint line above the site of pathologic fracture; an intraarticular excision was done, removing the entire distal femur with wide margins.

As expandable tumor endoprostheses were not readily available, it was decided to fill in the resected defect with a temporary reconstruction. An improvised long stacked intramedullary Kuntscher nail $(32 \mathrm{~cm}$ long $11 \mathrm{~mm} \mathrm{~K}$-nail stacked onto a $26 \mathrm{~cm} 10 \mathrm{~mm} \mathrm{~K}$-nail welded together with a $12 \mathrm{~cm} 9 \mathrm{~mm}$ nail) was inserted retrograde with a cortical screw inserted into the clover-leaf open side of the nail in the remaining femur and proximal tibia to prevent rotation. The nail was wrapped with 2 packs of bone cement to simulate the removed femur (Figure 2). Accounting for the overlap of fragments due to the fracture, the actual bony defect would have been $28 \mathrm{~cm}$ but the bone cement reconstruction was only $25 \mathrm{~cm}$ due to the tightness of the shortened muscles, resulting in a leg length discrepancy (LLD) of $3 \mathrm{~cm}$ (Figure 3 ).

Tumor necrosis of the resected specimen was reported to be more than $90 \%$. The same chemotherapy regimen was continued, the last of 3 cycles given in March of 2009. The patient remained disease free and 6 months after the end of treatment, a Stanmore ${ }^{\circledR}$ invasive expandable endoprosthesis was inserted (Figure 4). At the time of reconstruction, the prosthesis was lengthened by $1 \mathrm{~cm}$, leaving a $2 \mathrm{~cm}$ leg lenth discrepancy. Over the course of the next 4-1/2 years, the patient underwent four more lengthenings for a maximium allowable expansion of $7 \mathrm{~cm}$ (Figure 5).

During this time, patient graduated from secondary school; he is now a senior College student in Computer Science. Despite a slight limp from both the LLD and the decreased muscle mass, the patient remained active, playing basketball against medical advice and attending regular body-building work-out sessions at the College Gym.

On latest follow-up August 2016, 8 years after biopsy and 20 years of age, the patient had a full range of knee motion, an LLD of less than $1 \mathrm{~cm}$, and a Musculoskeletal Tumor Society (MSTS) score of 28/30, receiving 1 point subtractions only on Activity Restriction and Gait (Figure 6). Other domains included in the scoring system on which the patient had a perfect score were pain, function, emotional acceptance and support.
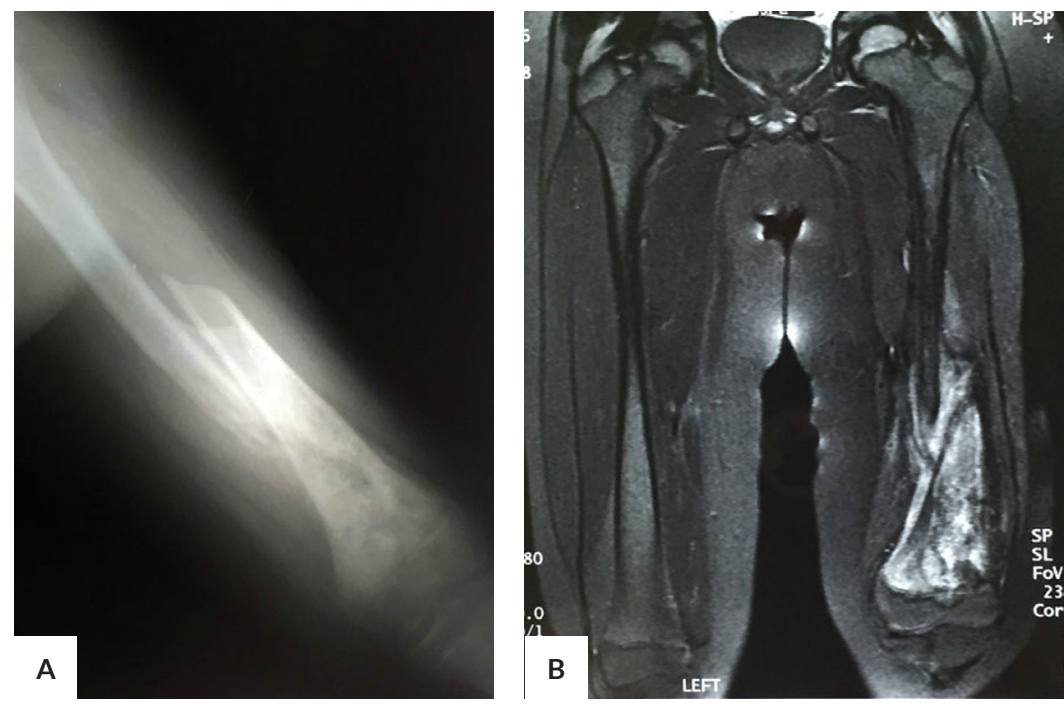

Figure $1 \mathrm{~A}$ and $1 \mathrm{~B}$. Plain radiograph and MRI showing osteosarcoma in the distal half of the femur with a pathologic fracture through the midshaft.

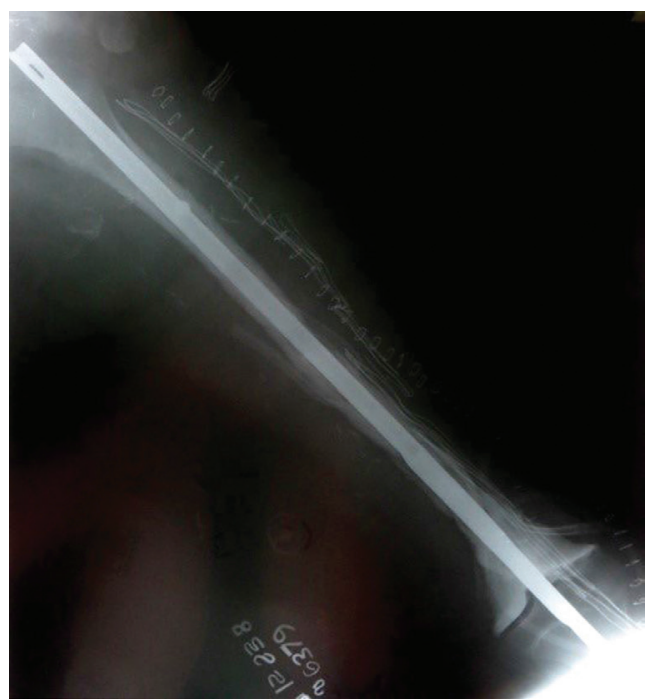

Figure 2. Plain radiograph after tumor resection, replacement with a bone cement spacer and fixation with an intramedullary rod (Kuntscher nail). 


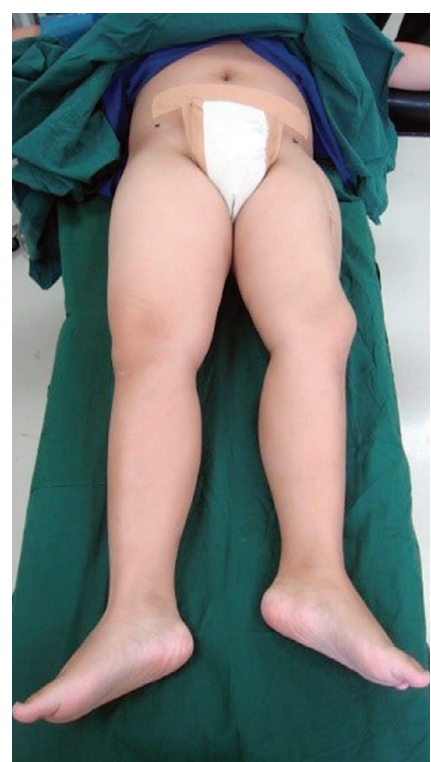

Figure 3. Clinical picture shows $3 \mathrm{~cm}$ leg length discrepancy.

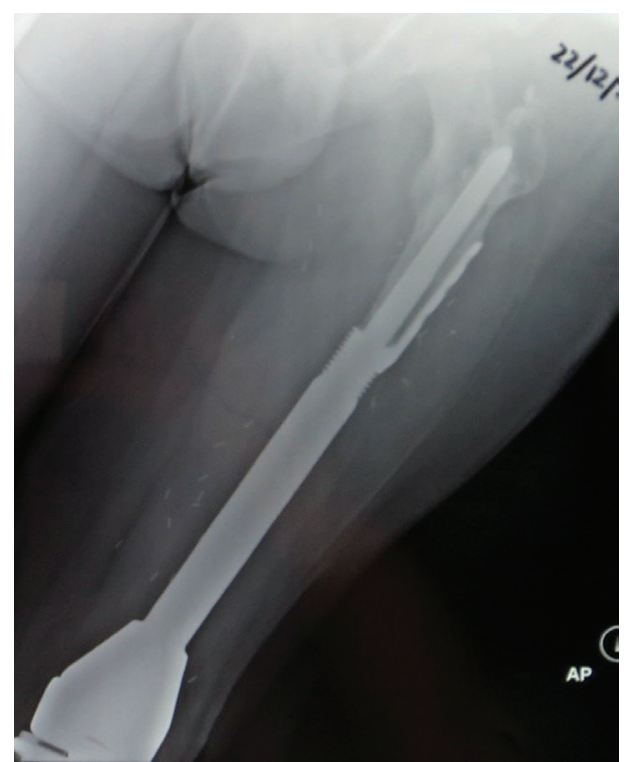

Figure 4. Plain radiograph after second operation shows Stanmore ${ }^{\circledR}$ expandable endoprosthesis replacing bone cement spacer and intramedullary rod.

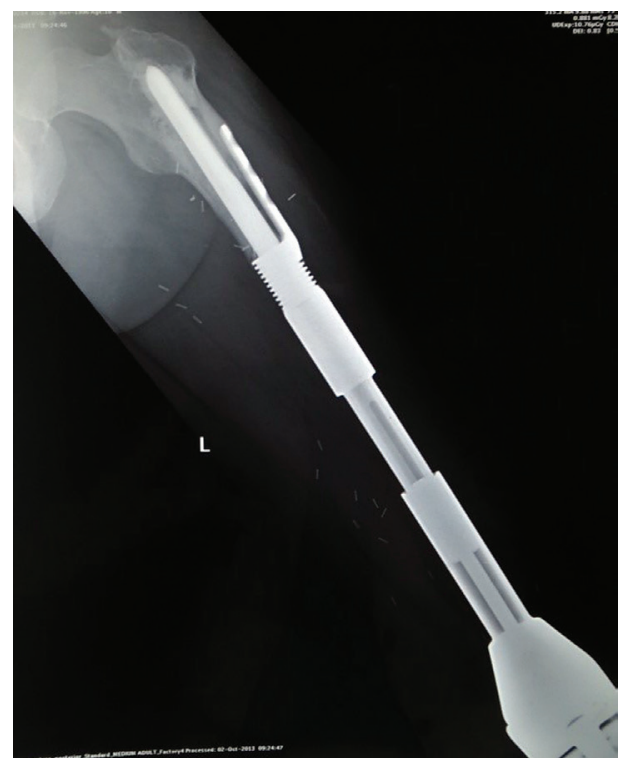

Figure 5. Plain radiograph showing expanded endoprosthesis. The endoprosthesis has been lengthened by $7 \mathrm{~cm}$ over a period of $4-1 / 2$ years.

\section{DISCUSSION}

Historically, a pediatric patient presenting with extremity high-grade osteosarcoma, complicated by a pathologic fracture, would have been offered amputation because of the fear of tumor contamination and the consequent local and systemic relapse. Not only would limb salvage have been considered more difficult in the smaller patient, but there were no viable solutions to the long term concern of severe limb length discrepancy. This, however, is not an absolute contraindication to limb salvage surgery but an appropriate patient selection must be done to ensure adequate local control. ${ }^{1}$ It has only been in recent years that tumor endoprostheses have become popular locally as a form of limb salvage reconstruction, although these prostheses,

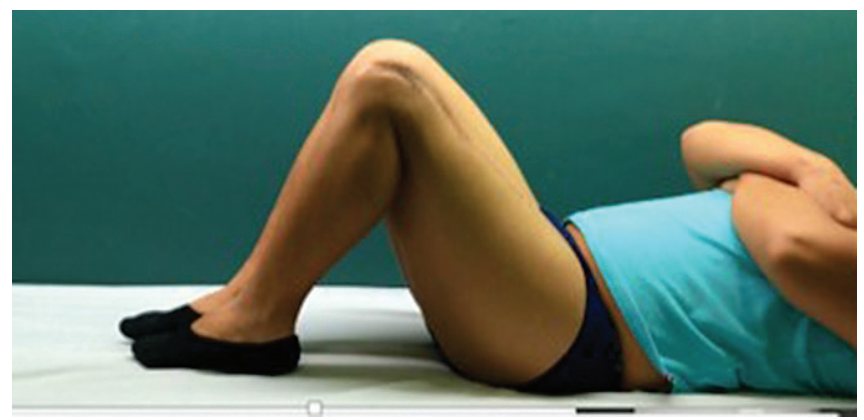

Figure 6. Clinical picture showing equal leg lengths on 8 year follow-up. Patient is 20 years old with full range of knee motion. especially expandable ones, are not readily available to patients and hospitals with relatively meager resources. This case is reported not because it is a rare diagnosis but because of what has been achieved through a combination of trailblazing, resourceful and innovative management in a patient who would otherwise have been amputated.

Deng, et al, in a recent collaborative study from 3 large Asian Orthopedic Oncology centers (China, India, Philippines), compared 95 patients with non-metastatic extremity osteosarcoma with a pathologic fracture with 887 non-metastatic osteosarcoma patients without a fracture. Limb salvage surgery (LSS) rate was $62.1 \%$ and $74.7 \%$, respectively and there was no significant difference in local recurrence rate and 5-year survival of the 2 groups. ${ }^{1}$ Our 12 year old patient showed a good response to neoadjuvant chemotherapy, allowing him to undergo limb salvage surgery despite his pathologic fracture. He is now 8 years since treatment and not only remains free of disease but also maintains a very active lifestyle.

Limb saving surgery, however, would not allow for a functional extremity unless the limbs are of fairly equal lengths. Reconstruction with the expandable type of prosthesis has the advantage of achieving not only a good range of motion but also limb length equality. ${ }^{2}$ In a 2015 report from the Rizzoli involving 32 pediatric patients reconstructed with expandable endoprostheses, Ruggieri et al reported that patients achieved a total lengthening of $28 \mathrm{~mm}$ in an average of approximately 3 procedures. MSTS score averaged $79 \%$ and survival of prostheses was $78 \%$ and $66 \%$ at 48 and 72 months, respectively. ${ }^{3}$ Schindler et al, 
on the other hand, reported on 12 patients reconstructed with the Stanmore ${ }^{\circledR}$ invasive expandable endoprosthesis. Total lengthening averaged $5.2 \mathrm{~cm}$ with a mean number of 4.3 operations; MSTS score was $77 \% .^{4}$ Our patient was lengthened $70 \mathrm{~mm}$ in 4 procedures over a period of $4-1 / 2$ years and final MSTS score was an excellent 93\%.

Reconstruction in our reported case, however, was achieved in 2 stages primarily because these implants were neither easily available nor readily affordable. Temporary reconstruction took the form of an improvised intramedullary Kuntscher nail wrapped with a bone cement spacer. In 2011 Puri et al reported 15 cases from a total of 818 limb sparing resections which were reconstructed with cement spacers. Two of these cases made use of a construct similar to ours: stacked Kuntscher nails with cement molded around the implant to bridge the bone defect. The constructs were also later converted to endoprostheses after 5 and 8 months, MSTS scores averaging $80 \% .{ }^{5}$ Our patient's construct was converted in 6 months without compromise to an eventual range of motion and function.

\section{CONCLUSION}

We report a pediatric patient with osteosarcoma, who contrary to traditional teaching and practice, received limb salvage surgery despite his age and presence of a pathologic fracture through his distal femoral osteosarcoma. He was reconstructed in 2 stages with an expandable tumor endoprosthesis, achieving $7 \mathrm{~cm}$ lengthening over a period of $4-1 / 2$ years. Today, 8 years since diagnosis and 2 years since lengthening, he is free of disease, has equal leg lengths, retains a full range of motion, and has an excellent MSTS score of 28/30 (93\%).

\section{Statement of Authorship}

All authors approved the final version submitted.

\section{Author Disclosure}

All authors declared no conflicts of interest.

\section{Funding Source}

This paper was funded by the authors.

\section{REFERENCES}

1. Deng ZP, Ding Y, Puri A, et. al. The surgical treatment and outcome of nonmetastatic extremity osteosarcoma with pathological fractures. Chin Med J (Engl). 2015;128(19):2605-8. doi: 10.4103/03666999.166025.

2. Jeys L, Abudu A, Grimer R. Expandable Prostheses. In: Malawer MM, Sugarbaker PH, eds. Musculoskeletal cancer surgery treatment of sarcomas and allied diseases. Dordrecht: Kluwer Academic Publishers; 2001:457-82.

3. Ruggieri P, Mavrogenis AF, Pala E, et.al. Outcome of expandable prosthesis in children. J Pediatr Orthop. 2013;33(3):244-53. doi: 10.1097/BPO.0b013e318286c178.

4. Schindler OS1, Cannon SR, Briggs TW, Blunn GW. Stanmore custom-made extendible distal femoral replacements. J Bone Joint Surg Br. 1997;79(6):927-37.

5. Puri A, Gulia A, Pruthi M, Koushik S. Primary cement spacers: a cost-effective, durable limb salvage option for knee tumors. Knee. 2012(4):320-3. doi: 10.1016/j.knee.2011.06.012.
Have you read the current trends in Medical and Health Research in the Philippines?

\section{Acta Medica Philippina The National Health Science Journal}

Access Online: www.actamedicaphilippina.org 